\title{
A Differential Time-of-flight Spectrometer of Very Slow Neutrons
}

\author{
Yu. N. Pokotilovski ${ }^{1}$ \\ Joint Institute for Nuclear Research \\ 141980 Dubna, Moscow region, Russia \\ M. I. Novopoltsev \\ Mordovian State University \\ 430000 Saransk, Russia \\ P. Geltenbort and Th. Brenner \\ Institut Laue-Langevin, \\ BP 156, 38042 Cedex 9 Grenoble, France
}

\begin{abstract}
A time-of-flight spectrometer of neutrons in the energy range (0.052.5) $\mu \mathrm{eV}$ is described. This spectrometer has been tested my measuring the total and differential neutron cross sections for a number of materials: $\mathrm{Al}, \mathrm{Cu},{ }^{6} \mathrm{LiF}, \mathrm{Si}, \mathrm{Zr}$, teflon, polyethylene and liquid fluoropolymers, that are essential for experiments in the physics of ultracold neutrons.
\end{abstract}

PACS: 28.20.-v; 28.20.Cz; 29.30.Hs;

Keywords: Ultracold neutrons; Total cross section; Elastic neutron scattering

\section{Introduction}

Ultracold neutrons (UCN) [1] with energy less than $\sim 0.25 \mu \mathrm{eV}$ can be confined within closed volumes over periods of time as long as the neutron $\beta$-decay. This phenomenon has found well known applications in the investigations of fundamental properties of the neutron [2]: searching for the neutron electric dipole moment, measuring the neutron lifetime, investigation of angular correlations in neutron decay. The examples of applications of very low energy neutrons in the $\mu \mathrm{eV}$ energy range and lower for studies in condensed matter physics are rather scarce (see for example the reviews [3, 4]).

Owing to the very low neutron energy it is possible to reach rather high energy and momentum resolution: $\sim 1 \mathrm{neV}$ and $\sim 10^{-4} \AA^{-1}$ respectively.

This high resolution opens up the obvious new possibility in investigations of supramolecular structure and dynamics of matter due to high sensitivity of very low energy neutrons to scattering on the objects of the size 10-1000 A. The latter is particularly interesting foe studying in the soft matter, in physics of polymer and biological science, where slow motion of large molecules and clusters present one of most interesting and poorly investigated domains.

The only reason for such a modest scale of application of very low energy neutrons in condensed matter research is low intensity of neutron flux in the very low energy range.

The best up to now source of neutrons in this energy range - ILL UCN turbine [5] yields $\sim 50 \mathrm{n} /\left(\mathrm{cm}^{2} \mathrm{~s} \mathrm{neV}\right)$ in the maximum of the neutron spectrum in vicinity $750 \mathrm{neV}(\mathrm{v} \approx 12 \mathrm{~m} / \mathrm{s})$ and the UCN density in the storage mode $\sim 40 \mathrm{~cm}^{-3}$.

${ }^{1}$ Corresponding author; e-mail: pokot@nf.jinr.ru 
Last years brought hope for significant progress in intensity of very low energy neutron beams after commissioning of new UCN sources [6, 7, 8]. It is connected with possible use of the most effective cold moderators: solid deuterium [9] or solid deuterocarbons at low temperatures $\sim 5-10 \mathrm{~K}$ for production of very cold neutrons.

\section{Description of the spectrometer}

As applied to UCN, the necessity to have a neutron guide tube in the flight path in order to prevent neutrons from falling in the gravitational field (on a 1-m-long flight path, a neutron with velocity $5 \mathrm{~m} / \mathrm{s}$ falls down in the gravitational field by $20 \mathrm{~cm}$ ) is a significant drawback of time-of-flight neutron spectrometers with a horizontal location of the flight path [10].

Nonmirror neutron reflection from the surface, proceeding with a some probability even in the best mirror neutron guide tubes, changes the measured longitudinal component of the neutron velocity. Moreover, in relatively long neutron guides with a large number of neutron reflections from the walls of the neutron guide tubes on the path from the chopper to the detector, diffuse scattering of a portion of neutrons causes the neutron guide tube to act as a storage chamber. This phenomenon is intensified by the fact that neutrons with lowest energies are reflected from the surface of the detector entrance windows. All these facts result in hold-up of scattered neutrons and distortions in the measured spectrum. With this in mind we used previously in the flight path of the time-of-flight spectrometers with a horizontal neutron guide the shortest possible neutron guide tubes $(<50 \mathrm{~cm}$ long), a special nonreflecting neutron detectors, and the fastest admissible for the UCN beam modulation in order to attain an acceptable resolution of 2-3 neV [11].

In the described here spectrometer, the flight path has a vertical geometry, and the large area detector is located in a horizontal plane, so that the neutron guide tube is needless. Neutrons are accelerated on the flight path between the sample and the detector in the gravitational field; as a result, the probability that a neutron will be reflected from the surface of the detector's entrance window becomes almost zero.

By contrast to the vertical time-of-flight spectrometers of very slow neutrons with the flight path located ahead of the sample [12, 13], the flight path of our spectrometer is disposed past the sample. This allows us to measure angular distributions of scattered neutrons and, hypothetically in future, measure doubly differential cross sections $d^{2} \sigma / d \Omega d \epsilon$, provided that the neutron beam incident on the sample is monochromatized. The axial geometry with respect to the vector of the gravitational field permits the use of a coordinate detector for meausuring, without distortions, the angular distribution of scattering of the collimated neutron beam.

The angular distribution of scattered neutrons must also be monitored in measurements of the total cross sections, in which the elastic cross section component of the total cross section should be separated from the inelastic component: at very low energies, elastic scattering basically proceeds on inhomogeneities and can be distinguished in angular distributions.

The schematic diagram of the spectrometer is shown in Fig. 1.

Neutrons travel over neutron guide tube 1 , which is bent through $90^{\circ}$, toward collimator 2 and then to chopper 3 of the collimated beam. Additional collimation of the neutron beam is effected by collimator 4 located past the chopper. Samples 11 under investigation are located in sample holder 5 inside the vacuum chamber and can be cooled to low temperatures by pumping liquid nitrogen through the sample holder. The sample holder parts shown in the inset in Fig. 1 are used for better maintenance of the temperature conditions. Scattered neutrons that passed the sample enter vertical evacuated flight path 7 and move in parabolic trajectories 8 in the Earth's gravitational field. The axis of these parabolic trajectories coincides with the vertical axis of the ring detector.

Neutrons are detected by gas-filled coordinate annular detector 9 , which is located in a horizontal plane and has ring geometry (the radial width of the ring counters is $3.4 \mathrm{~cm}$, and maximum radius is $29 \mathrm{~cm}$ ). The vertical distance from the chopper to the detector plane may be $79 \mathrm{~cm}$ or $160 \mathrm{~cm}$, however the latter variant has not yet been tested. 


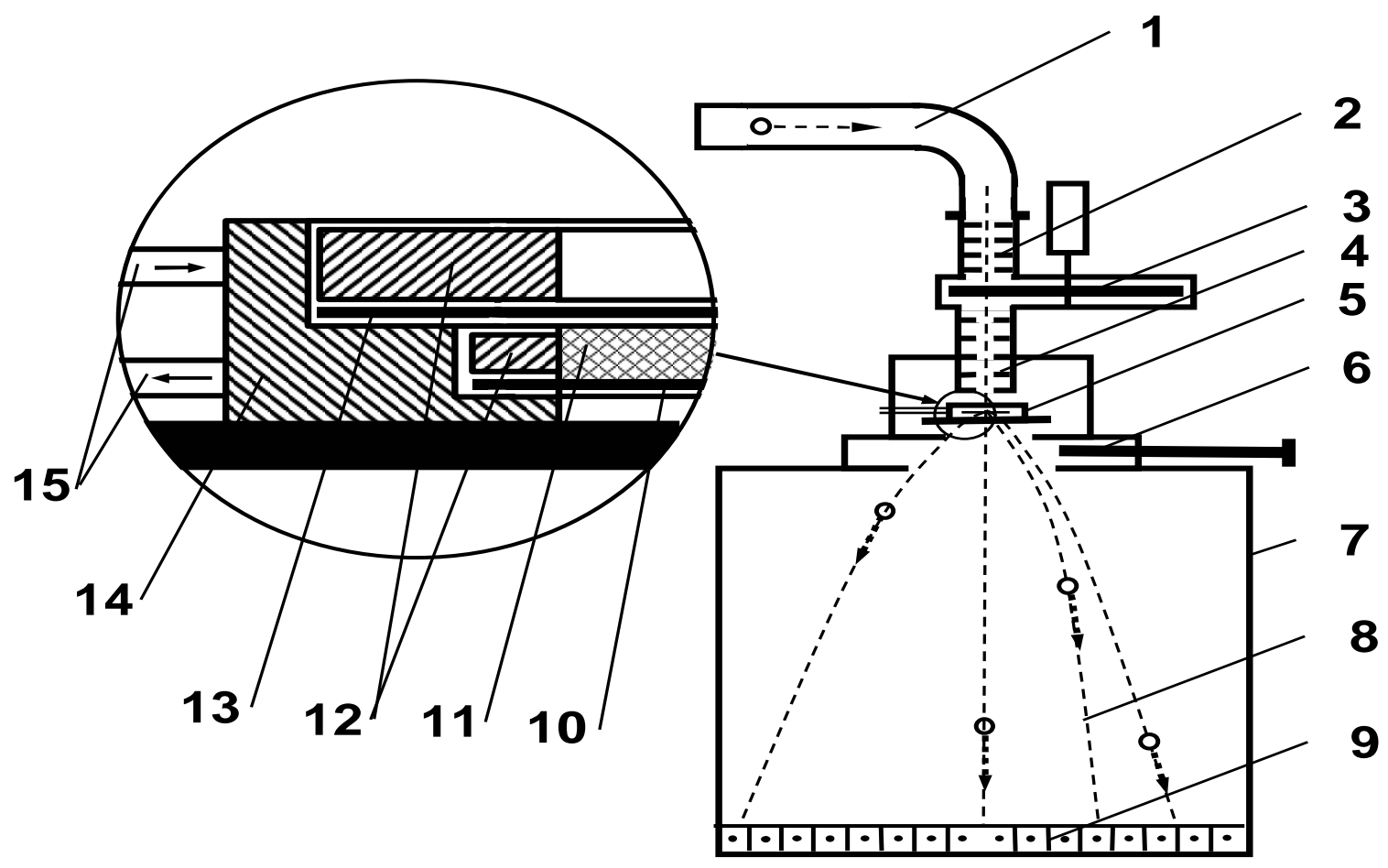

Figure 1: The scheme of the spectrometer: 1 - neutron guide, 2 and 4 - collimators, 3 - chopper, 5 - sample holder, 6 - VAT valve, 7 - vacuum barrel, 8 - neutron trajectories, 9 - annular neutron position sensitive detector, 10 and 13 - Si plates, 11 - sample, 12 - copper rings, 14 - copper holder, 15 - liquid nitrogen. 
Between the vacuum time-of-flight barrel 7 and the sample chamber 5 , there is large-diameter vacuum gate valve 6 (VAT DN 160) used to replace samples without putting atmospheric air into the barrel.

The replaceable chopper discs of $42.8 \mathrm{~cm}$ in diameter are made of $3 \mathrm{~mm}$-thick polyvinylchlorid coated with a gadolinium-containing paint. The chopper rotation frequency may varied from 3 to $8 \mathrm{~Hz}$ and is electronically stabilized with precision $\sim 2 \times 10^{-4}$. The chopper is driven by a SP-361 dc motor via a magnetic clutch. The trapezoidal windows in the chopper discs are 2,4 and $8 \mathrm{~cm}$ wide, wich corresponds to $\sim 2,4$ and $8 \%$ of the open beam time with respect to the chopper rotation period.

A start signal from the chopper is produced by a LED via a slit drilled in the disc on the side opposite to the chopper window.

The 8-ring coordinate detector has a thickness of $1 \mathrm{~cm}$ and is filled with a mixture of $50 \%{ }^{3} \mathrm{He}$ and $50 \% \mathrm{CF}_{4}$ (we used ${ }^{10} \mathrm{BF}_{3}$ gas at the first stage) to a pressure of $\sim 100$ mbar. This corresponds to $n \sigma \approx 1.5$ for neutrons with velocity $10 \mathrm{~m} / \mathrm{s}$.

A $100-\mu \mathrm{m}$-thick aluminium foil supported by stainless steel grid is used as a membrane of the entrance window of the coordinate detector. The inner surface of the time-of-flight volume and the surface of the stainless steel grid are covered with polyethylene to prevent neutrons with very low energies from being reflected.

This spectrometer has been used to measure the total and differential neutron cross sections. In the future, it is expected that doubly differential cross sections $d^{2} \sigma / \mathrm{d} \Omega d \epsilon$ will be measured.

From the obvious relations for vertical and horizontal velocity components

$$
v_{\text {vert }}=\frac{h}{t}-\frac{g t}{2}, \quad v_{\text {hor }}=\frac{r}{t}
$$

where $h$ is the vertical distance between the planes of the chopper and the detector, $t$ is the neutron time-offlight, $r$ is the radial coordinate of the neutron detection point, and $g$ is the gravitational acceleration, it is easy to obtain:

$$
v=\left(\frac{r^{2}+h^{2}}{t^{2}}-g h+\left(\frac{g t^{2}}{2}\right)^{2}\right)^{1 / 2}
$$

The uncertainties in velocities depending on uncertainties in $h, t$ and $r$, are

$$
\Delta v_{h}=\frac{2 h-g t^{2}}{2 v t^{2}} \Delta h, \quad \Delta v_{t}=\frac{\left(g t^{2}\right)^{2}-4\left(r^{2}+h^{2}\right)}{4 v t^{3}} \Delta t, \quad \Delta v_{r}=\frac{r}{v t^{2}} \Delta r .
$$

The error in determining the horizontal coordinate is determined by detector ring width $\Delta R$ and the uncertainty due to angular spread $\Delta \theta$ of the incident neutron beam, which is equal to $\Delta \theta\left(r^{2}+h^{2}\right) / h$.

The uncertainties in the neutron energy are shown in Fig. 2 at $\Delta t=3 \mathrm{~ms}$ and $6 \mathrm{~ms}, h=79 \mathrm{~cm}, \Delta h=0.5 \mathrm{~cm}$ and $\Delta r=1.7 \mathrm{~cm}$. These uncertainties are weakly dependent on the radius of the detector $\mathrm{R}$. The main contribution to these uncertainties comes from $\Delta t$.

The expressions for the neutron wave vector transfer $Q$ with account of gravity are:

$$
\sin \theta=\frac{r}{v t}, \quad Q=2 k \cdot \sin (\theta / 2),
$$

$k\left(\AA^{-1}\right)=1.588 \times 10^{-5} v(\mathrm{~cm} / \mathrm{s})$. The expressions for the uncertainties in $\mathrm{Q}$, which include angle of divergence of the incident beam $\Delta \theta$, and the uncertainties in $h, t$, and $r$ are rather sophisticated and are not shown here. The calculated energy dependence of $\mathrm{Q}$ and $\Delta Q$ are shown in Fig. 3 for two values of radii of the neutron detector rings $R$ of $6.6 \mathrm{~cm}$ and $27 \mathrm{~cm}, \mathrm{~h}=79 \mathrm{~cm}$ and $\Delta t=3 \mathrm{~ms}$.

The tests included investigation of the properties of position sensitive gas neutron detector filled with different gases: ${ }^{10} \mathrm{BF}_{3}$ and a mixture of ${ }^{3} \mathrm{He}$ and $\mathrm{CF}_{4}$, optimization of the neutron collimators and test measurements of the total and differential cross section for several samples. On-line monitoring and recording of pulse-hight spectra from all rings of the detector was carried out in all measurements. Minimization of the background and optimization of the detector radiation shielding under conditions of a low neutron flux were also essential. 


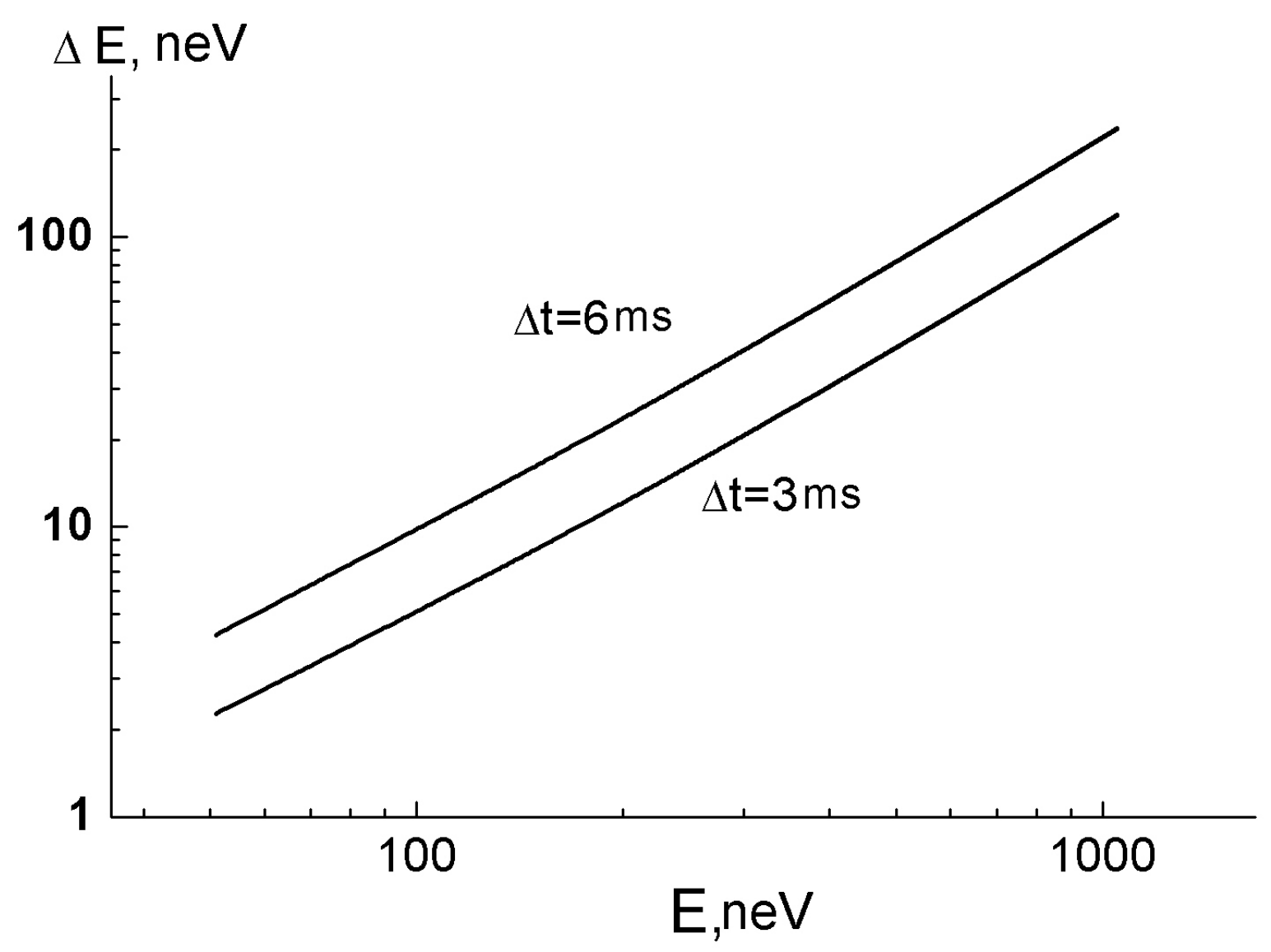

Figure 2: Calculated energy resolution of the spectrometer at a flight path length of $79 \mathrm{~cm}$.

The total and differential cross sections were measured with rather good (for this energy range) collimation of the incident beam, since, at collimation such as this, the influence of the sample structure manifests itself to the maximum degree. All the measured samples are relevant to the experiments in UCN physics and therefore, a matter of some practical interest. The $\mathrm{Al}$ and $\mathrm{Zr}$ foils are used as entrance windows in UCN detectors, the exit windows in the neutron sources, and, sometimes, as energy filters of neutrons in some specific experiments with UCN. Polyethylene is used as an "ideal" UCN absorber, in particular, in gravitation UCN spectrometers [14, 15] and for calibration of UCN losses in the UCN storage chambers. Fluoropolymers are now thought to be the best coatings for walls of UCN storage chambers [16]. ${ }^{6} \mathrm{LiF}$ layers are used in some UCN detectors [17]. Being a very homogeneous material, silicon single-crystal wafers presented our special interest and have found applications in our experiments with very cold neutrons. Their good feature is that they do not broaden the collimated neutron beam and are therefore suitable for sample chambers in neutron scattering experiments.

\section{Measurements and results}

The measured scattering cross sections of very slow neutrons are shown in Figs. 4-7. Figs. 4 and 5 illustrate the transmission of neutrons through an inhomogeneous polycrystalline medium.

The transmission of slow neutrons through inhomogeneous media was investigated earlier in [18]. It was 


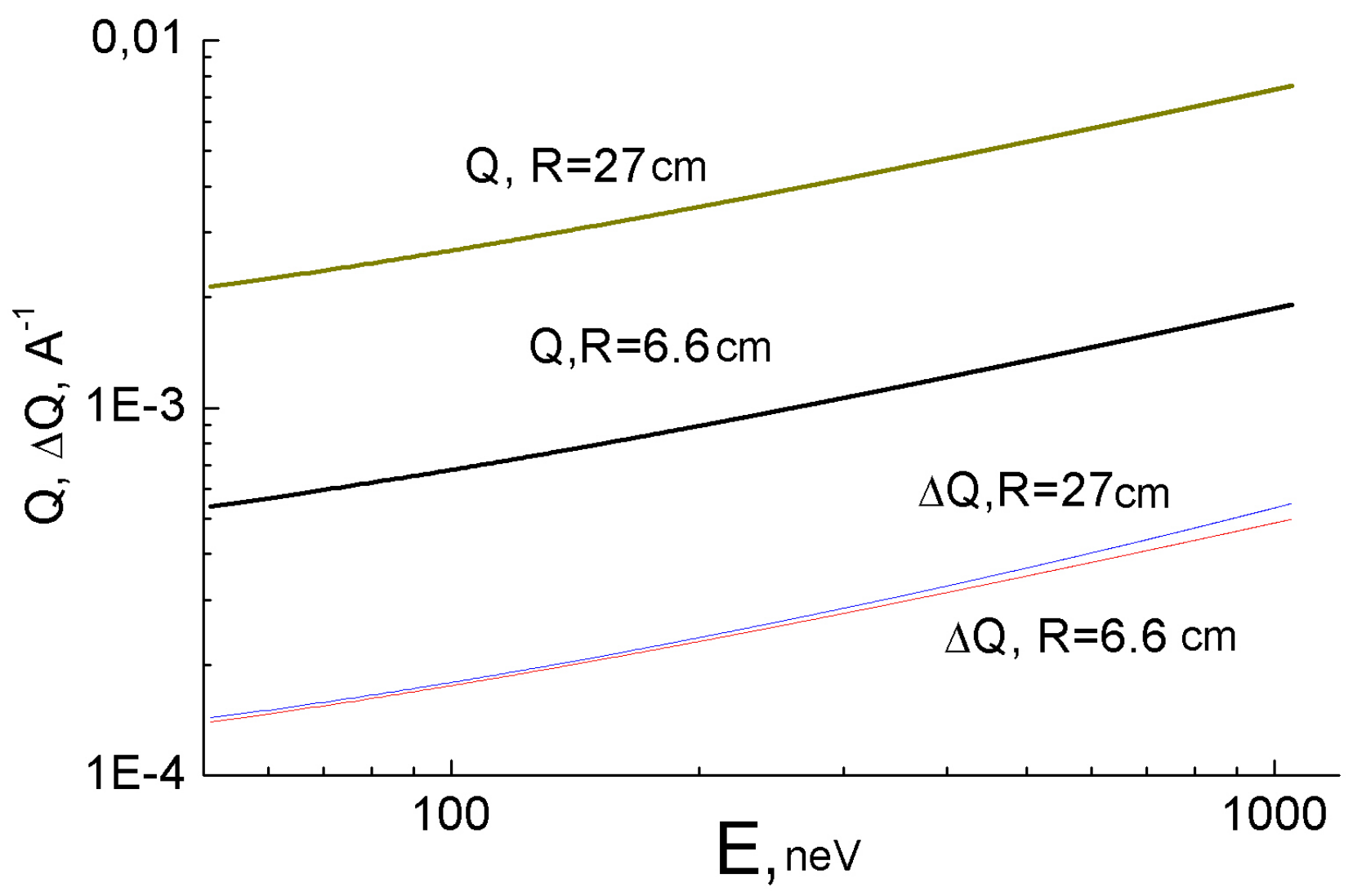

Figure 3: Calculated resolution in transmitted wave vector $Q$ (solid lines) and $\Delta Q$ (dashed lines) at $\Delta t=3 \mathrm{~ms}$ and a flight path length of $79 \mathrm{~cm}$.

shown that, based on the transmission as a function of the neutron wave length, one can deduce characteristic parameters of inhomogeneities, in particular their size and density. Our measurements were performed at large wavelengths, up to $\sim 10^{3} \AA$, for which the sensitivity to scattering on inhomogeneities and clusters is substantially higher and the measured cross sections are several orders of magnitude greater than the cross sections extrapolated from the energy range of cold neutrons according to the $1 / v$ law.

It is known that in the Born approximation the differential macroscopic cross section for elastic scattering of neutrons passing through an isotropic inhomogeneous medium has the form [19]:

$$
\frac{d \Sigma_{e l}}{d \Omega}=\frac{1}{\pi}\left(\frac{m}{\hbar^{2}}\right)^{2} \int_{0}^{\infty} G(\rho) \frac{\sin (q \rho)}{q \rho} \rho^{2} d \rho,
$$

where $m$ is the neutron mass, $q=\left|\vec{k}^{\prime}-\vec{k}\right|$ is the change of the neutron wave vector upon scattering, and $G\left(\vec{r}, \vec{r}^{\prime}\right)=<\delta U(\vec{r}) \delta U\left(\vec{r}^{\prime}\right)>$, is the correlation function of fluctuations of the local neutron-medium interaction potential, and $\left(\rho=\left|\vec{r}^{\prime}-\vec{r}\right|\right)$.

This potential is

$$
U=\frac{\hbar^{2}}{2 m} \sum_{i} 4 \pi N_{i} b_{i}
$$




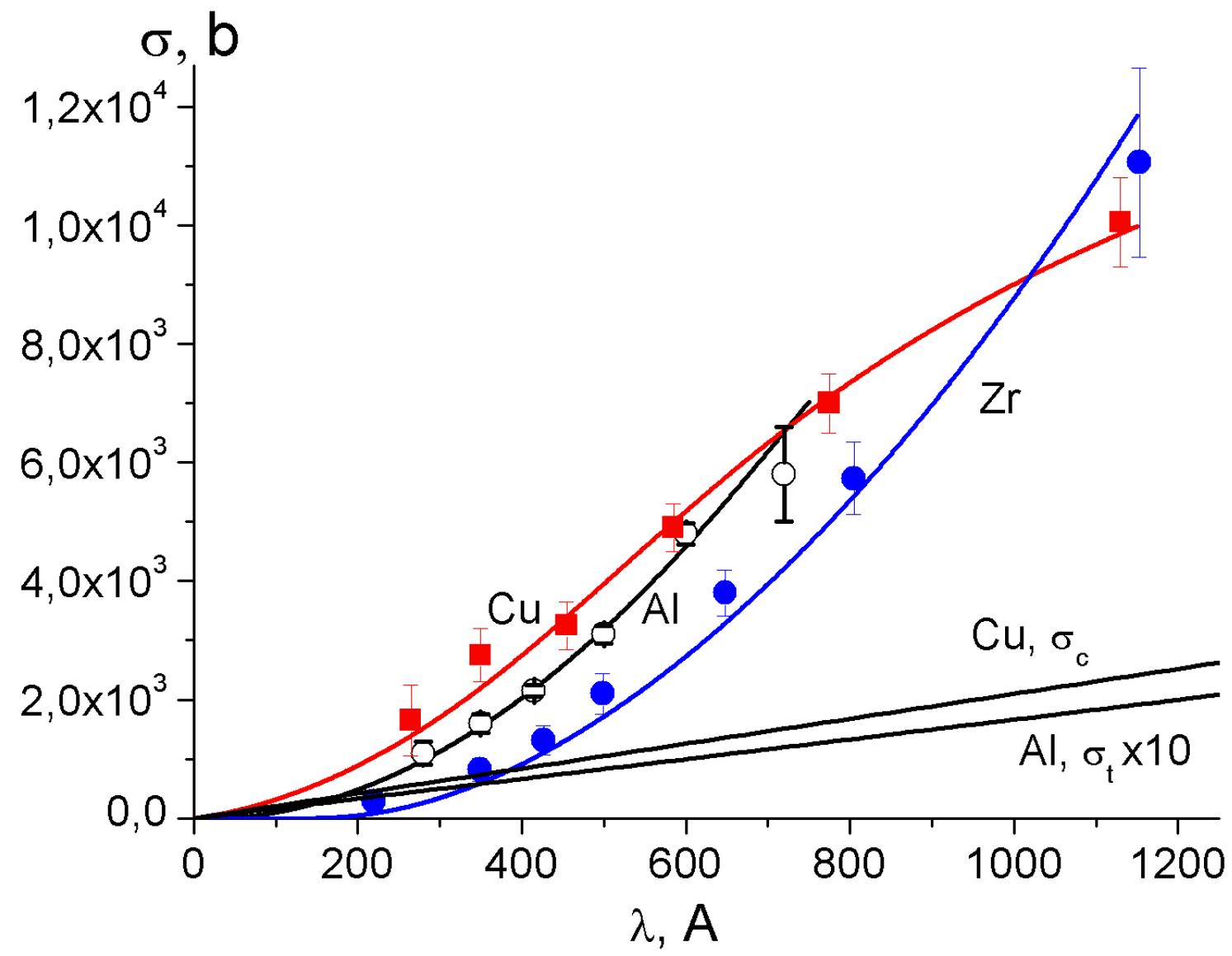

Figure 4: Total cross sections for $\mathrm{Al}$ (Goodfellow, 99.99\%, not annealed), $\mathrm{Cu}$ (Goodfellow, 99\%, annealed), and $\mathrm{Zr}$ (Goodfellow, 99.8\%, $50 \mu \mathrm{m}$, not annealed) foils. The straight lines are the result of extrapolation of cross section from the region of cold energies according to $1 / v$ law for $\mathrm{Al}\left(\sigma_{t o t}=1.8 \mathrm{~b}\right.$ at an energy 0.6 mev [23], neutron capture cross section for $\mathrm{Cu}$ [23] and cross section for $\mathrm{Zr}\left(\sigma_{t o t}=1.45 \mathrm{~b}\right.$ at the energy $\left.1 \mathrm{mev}\right)$ [23]. The curves passing through the experimental points is result of fitting for $\mathrm{Al}$ according to Eq. (12) with $G_{0}=(6.5 \pm 2.7) n e V^{2}, \rho_{0}=(456 \pm 190) \AA$, for $\mathrm{Cu}$ according to Eq. (12) with $G_{0}=(13 \pm 3) n e V^{2}, \rho_{0}=(294 \pm 35) \AA$, and for $\mathrm{Zr}$ according to Eq. (9) with $G_{0}=(1.9 \pm 0.12) n e V^{2}, \rho_{0}=(840 \pm 95) \AA$. 


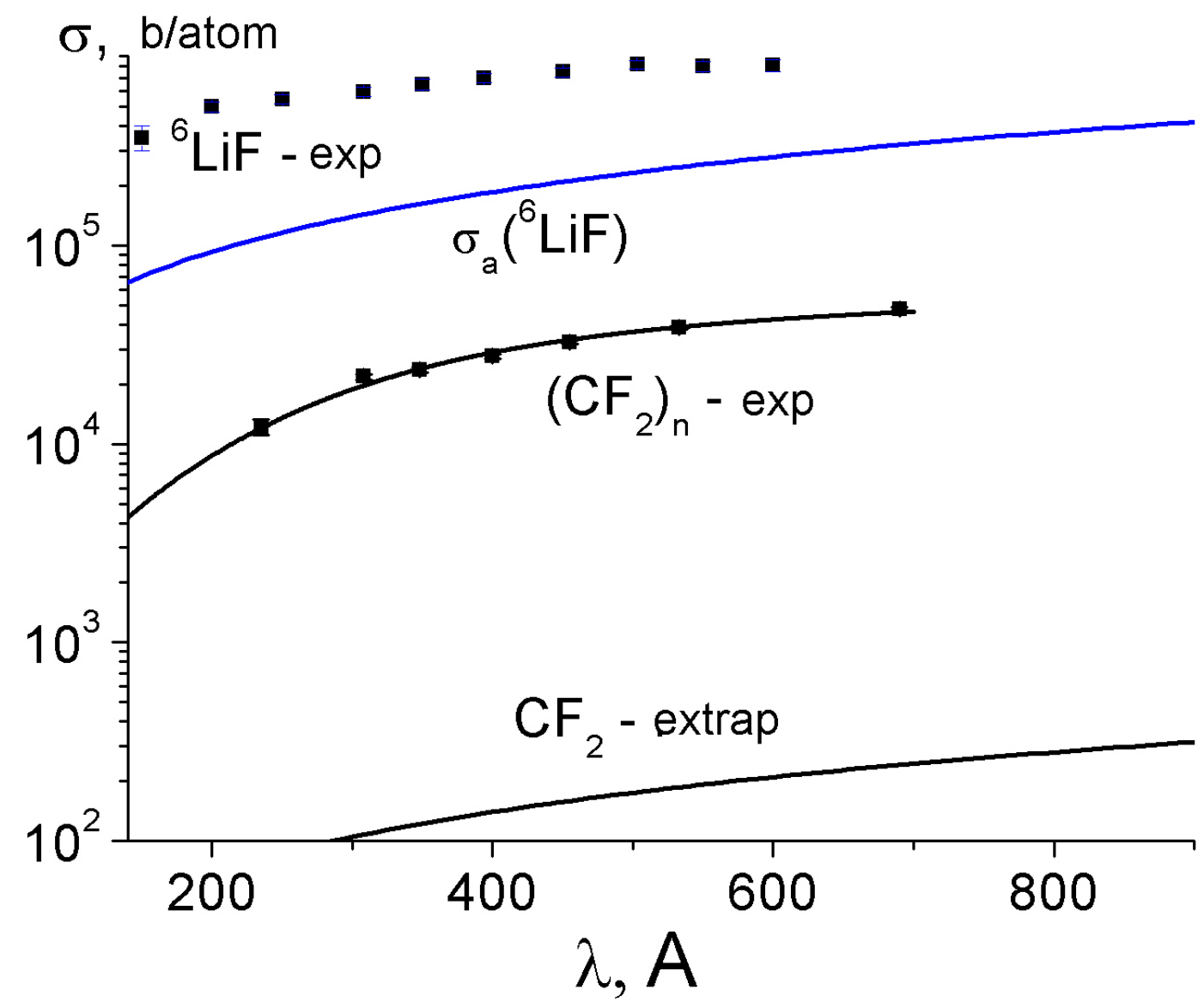

Figure 5: The total cross section for the Teflon film $10 \mu$ m thick and ${ }^{6} \mathrm{LiF}$ layer $240 \mu \mathrm{g} / \mathrm{cm}^{2}$ thick. The solid lines are the results of the extrapolation of the cross section for Teflon from the region of cold energies according to the $1 / v$ law with $\sigma_{\text {tot }}=5.18 \mathrm{~b} /$ atom for an energy $0.376 \mathrm{mev}$ [21] , capture cross section for ${ }^{6} \mathrm{LiF}$, and the results of fitting of the experimental points for Teflon according to Eq. (12) with $G_{0}=(400 \pm 25) n e V^{2}, \rho_{0}=(170 \pm 15) \AA$. 
where $N_{i}$ is the atomic density and $b_{i}$ is coherent scattering length of nuclei in the medium, so that $\delta U(\vec{r})=$ $U(\vec{r})-<U(\vec{r})>$.

After integrating over the solid angle with due account of the solid angle of the neutron detector the total cross section is presented as:

$$
\Sigma_{e l}(k)=2\left(\frac{m}{\hbar^{2}}\right)^{2} \frac{1}{k^{2}} \int_{0}^{\infty} G(\rho)\left[\cos \left(2 k \rho \cdot \sin \theta_{0}\right)-\cos (2 k \rho)\right] d \rho,
$$

where $2 \theta_{0}$-is the angle with which the neutron detector is viewed from the sample.

For the exponential correlation function

$$
G(\rho)=G(0) e^{-\rho / \rho_{0}},
$$

where $\rho_{0}$ is the correlation length, the expression for the total cross section is

$$
\Sigma_{e l}(k)=2\left(\frac{m}{\hbar^{2}}\right)^{2} \frac{G_{0} \rho_{0}}{k^{2}}\left[\frac{1}{1+4 k^{2} \rho_{0}^{2} \sin ^{2} \theta_{0}}-\frac{1}{1+4 k^{2} \rho_{0}^{2}}\right],
$$

and the differential cross section has the form

$$
\frac{d \Sigma_{e l}}{d q}=\frac{2 \pi q}{k^{2}} \frac{d \Sigma}{d \Omega}=\frac{4 q}{k^{2}}\left(\frac{m}{\hbar^{2}}\right)^{2} \frac{G_{0} \rho_{0}^{3}}{\left(1+q^{2} \rho_{0}^{2}\right)^{2}} .
$$

For the Gaussian correlation function

$$
G(\rho)=G(0) e^{-\left(\rho / \rho_{0}\right)^{2}}
$$

the respective total cross section is

$$
\Sigma_{e l}(k)=\sqrt{\pi}\left(\frac{m}{\hbar^{2}}\right)^{2} \frac{G_{0} \rho_{0}}{k^{2}}\left[e^{-\left(k \rho_{0} \sin \theta_{0} / 2\right)^{2}}-e^{-\left(k \rho_{0} / 2\right)^{2}}\right]
$$

and the differential cross section is

$$
\frac{d \Sigma_{e l}}{d q}=\frac{2 \pi q}{k^{2}} \frac{d \Sigma}{d \Omega}=\frac{\sqrt{\pi}}{2}\left(\frac{m}{\hbar^{2}}\right)^{2} \frac{G_{0} \rho_{0}^{3} q}{k^{2}} e^{-\left(\rho_{0} q / 2\right)^{2}} .
$$

These formulas were used to interpret measurements of the total and differential cross sections for scattering from inhomogeneous samples (see also [18, 19, 20]). It has been determined in our work that, in some cases, one of the correlation functions (a Gaussian or exponential function) provides better agreement with the experimental data, but, in other cases, the difference in $\chi^{2}$ for these two models is inessential: both models represent experimental data well.

The following parameters of the correlation functions provide the best description of the measured cross sections:

$$
\begin{aligned}
& \mathrm{Al} \text { (Gaussian): } G_{0}=(6.5 \pm 2.7) n e V^{2}, \rho_{0}=(456 \pm 190) \AA ; \\
& \quad \text { Cu (Gaussian): } G_{0}=(13 \pm 3) n e V^{2}, \rho_{0}=(294 \pm 35) \AA ; \\
& \mathrm{Zr} \text { (exponential): } G_{0}=(1.9 \pm 0.12) n e V^{2}, \rho_{0}=(840 \pm 95) \AA ; \\
& \text { Teflon (Gaussian): } G_{0}=(400 \pm 25) n e V^{2}, \rho_{0}=(170 \pm 15) \AA .
\end{aligned}
$$

The total cross section of Teflon (Fig. 5) is two orders of magnitude larger than the total cross section extrapolated from the region of cold neutrons [21] and demonstrates the very high degree of inhomogeneity of this material.

Measuring the transmission of thin ${ }^{6} \mathrm{LiF}$ layers (with $90 \%{ }^{6} \mathrm{Li}$ content, we see that, even in this case, the total cross section is much greater than the extrapolated very large cross section for neutron capture by 


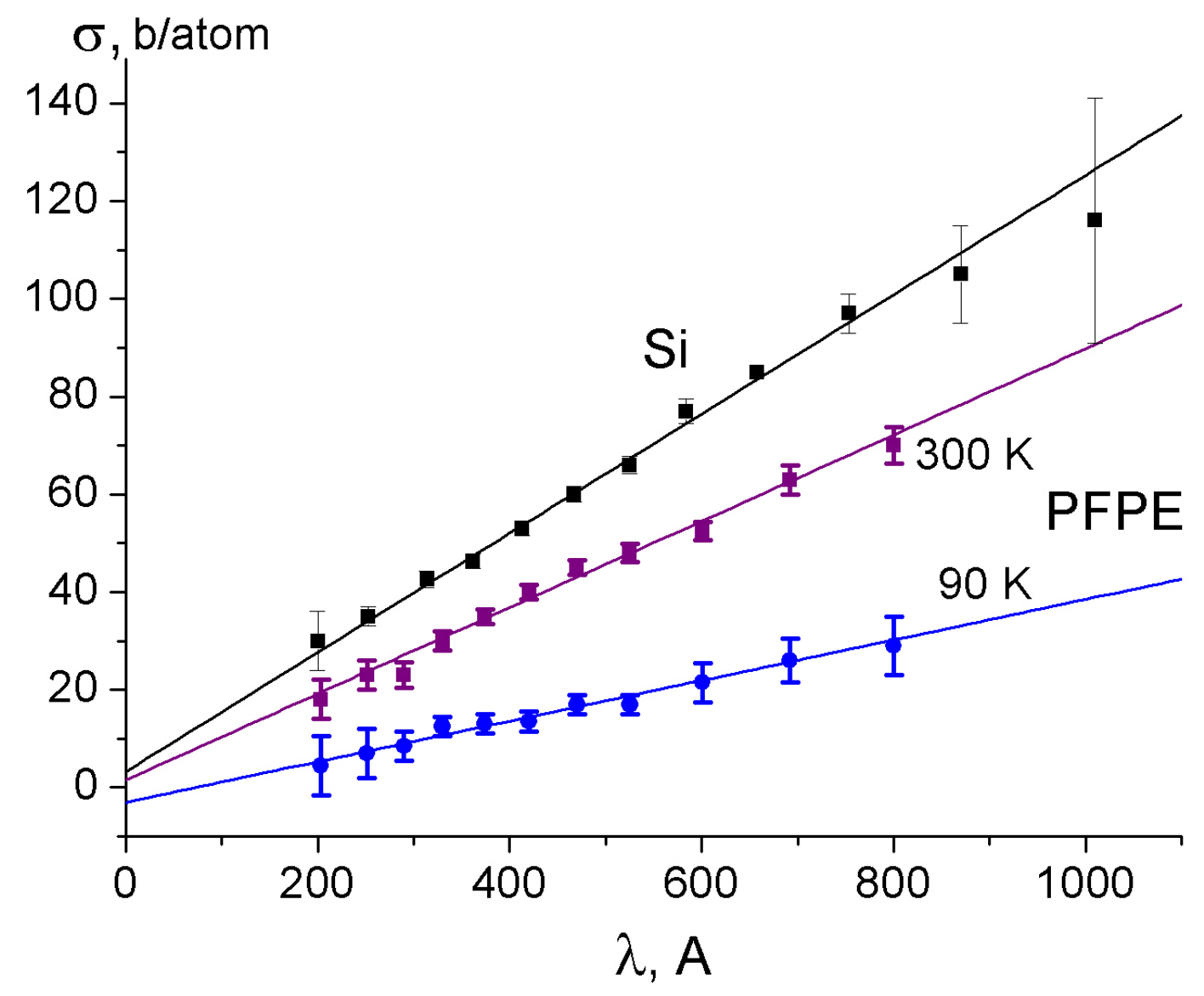

Figure 6: Total neutron cross sections for silicon and fluoropolymers. The straight lines passing through the experimental points are the fits according to Eq. (14) for silicon with $\sigma_{0}=$ $3.26 \pm 2.3) \mathrm{b}, a=(0.122 \pm 0.005) \mathrm{b} / \AA$, for liquid fluoropolymer at room temperature with $\left.\sigma_{0}=1.53 \pm 2.2\right) \mathrm{b}$ and $a=(8.83 \pm 0.5) \times 10^{-2} \mathrm{~b} / \AA$, and for the solid-state fluoropolymer at $\left.\mathrm{T}=90 \mathrm{~K} \sigma_{0}=3 \pm 3\right) \mathrm{b}$ and $a=(4.15 \pm 0.5) \times 10^{-2} \mathrm{~b} / \AA$. 


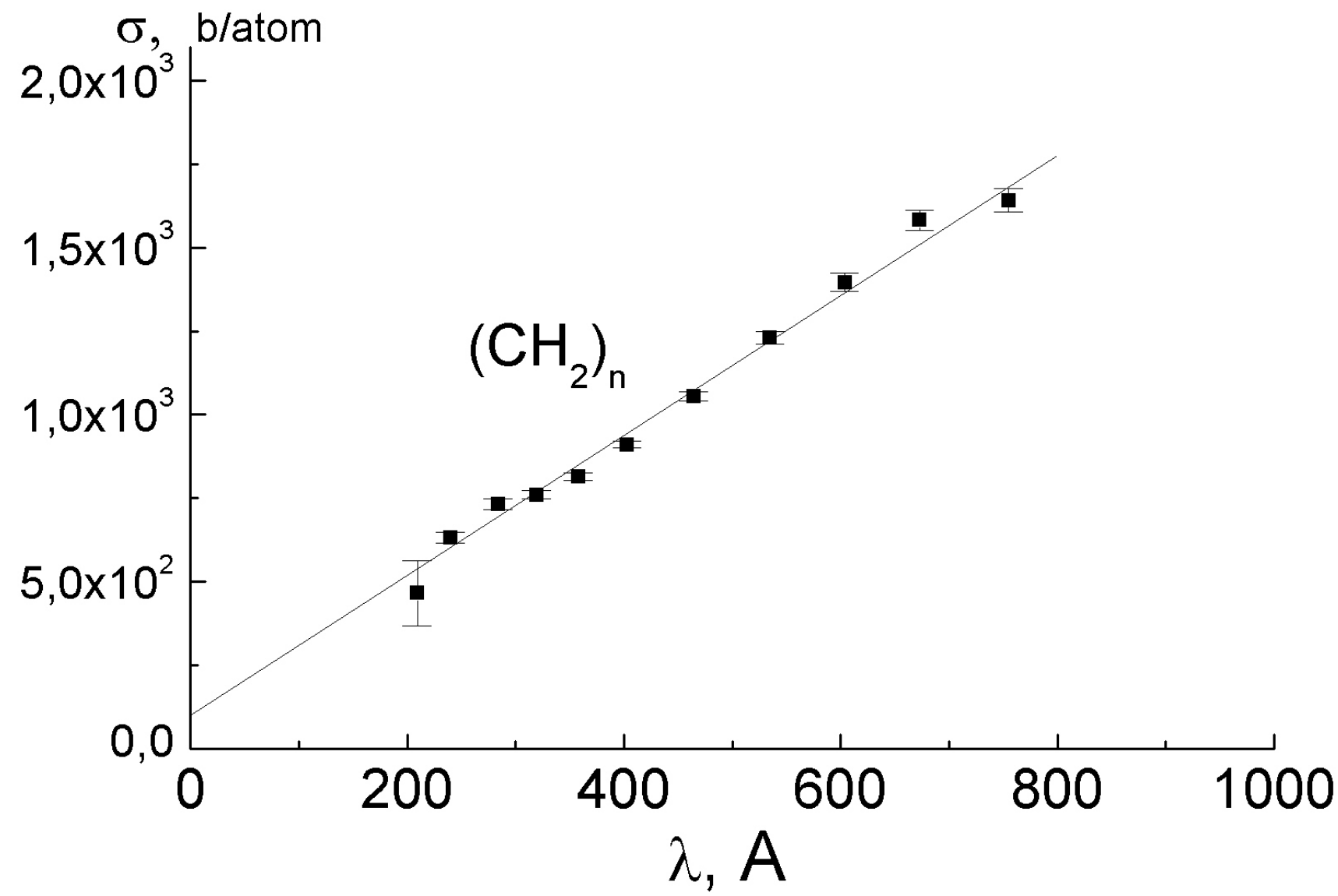

Figure 7: Total neutron cross section for the polyethylene film $7.88 \mathrm{mg} / \mathrm{cm}^{2}$ thick. The straight lines passing through the experimental points are the fits according to Eq. (14) with $\sigma_{0}=$ $96 \pm 18) \mathrm{b}, a=(2.07 \pm 0.04) \mathrm{b} / \AA \AA$.

${ }^{6} \mathrm{Li}$ nuclei (Fig. 5). This indicates that the ${ }^{6} \mathrm{LiF}$ layers obtained using the method of thermal evaporation in vacuum have a very high degree of inhomogeneity. Such a strong scattering from inhomogeneities will result in significant albedo of very slow neutrons and in a decrease in the UCN detector efficiency [17], particularly for neutrons near the potential barrier of the detector material.

Knowing parameters, characterizing the inhomogeneity of materials, one can calculate the transport of slow neutrons in the medium and the neutron albedo from this medium.

Figures. 6 and 7 demonstrate examples of homogeneous materials that do not show exhibit deviation from the inverse velocity law in their total cross sections.

The total cross section curves (Fig. 6) for Si-wafers and fluoropolymers at room temperature (liquid state) and low temperatures ( $90 \mathrm{~K}$, solid state) do not show any sign of inhomogeneities. The measurements of the angular distributions of neutron scattering for these materials have not demonstrated any broadening of the collimated UCN beam.

The total cross section of silicon (Fig. 6) is in good agreement with the cross section extrapolated from the 


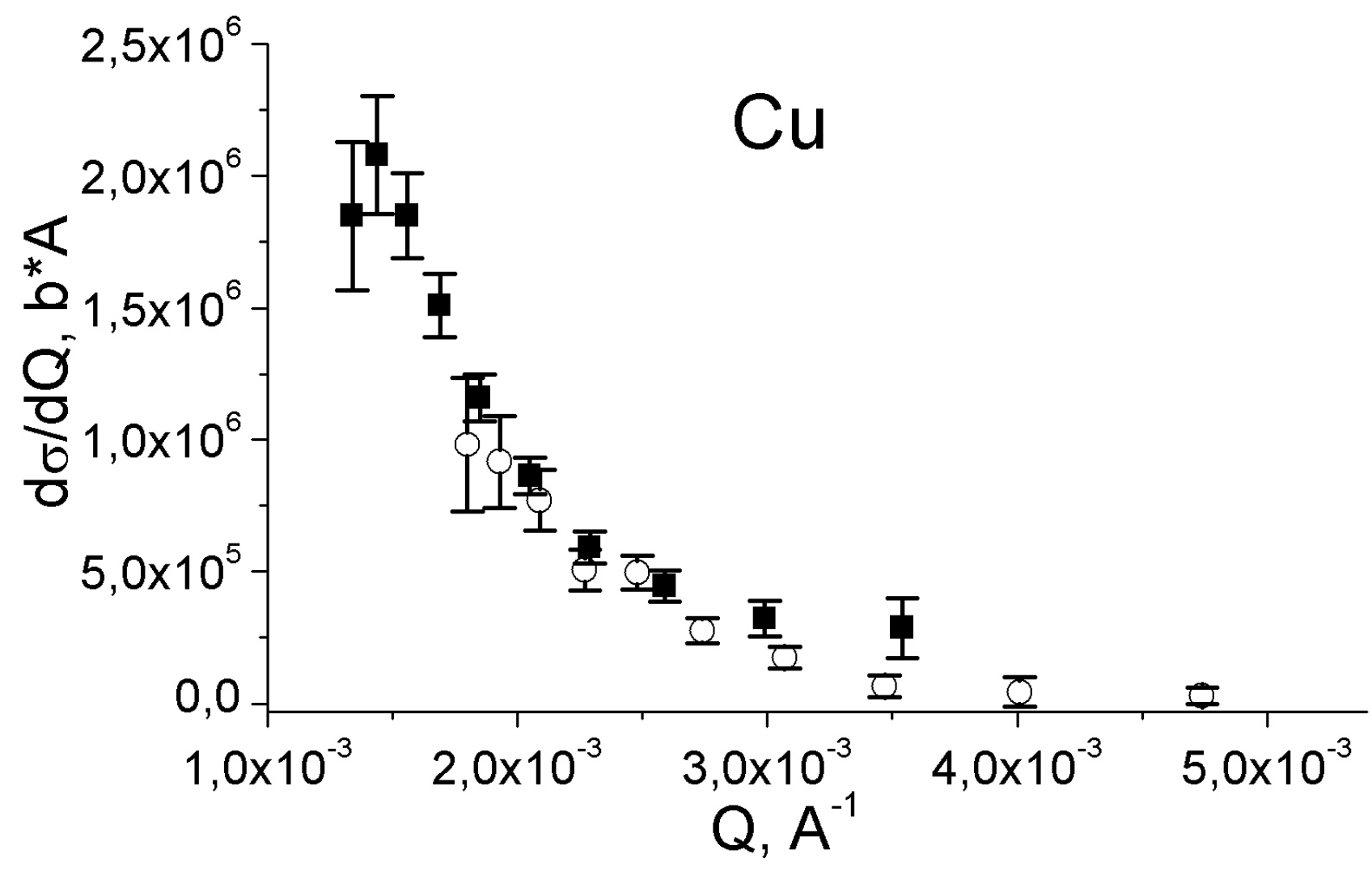

Figure 8: Differential cross section for copper. The squares and the circles correspond to two different detector rings.

energy range of cold neutrons [22, 23] according to $1 / v$ law and can be fitted by the dependence

$$
\sigma=\sigma_{0}+a \cdot \lambda^{\prime}
$$

where $\lambda^{\prime}$ is the neutron wavelength in the substance (corrected for the Fermi potential of the sample material), with parameters $\sigma_{0}=(3.26 \pm 2.3) \mathrm{b}, a=(0.122 \pm 0.005) \mathrm{b} / \AA$. This cross section is the sum of the energy-independent elastic coherent scattering and the cross section of neutron capture and heating, which are proportional to the neutron wavelength.

The low temperature liquid fluoropolymer POM-310 $0^{2}$ is the mixture of complex polyfluorooxymethylenes having the general formula $\mathrm{CF}_{3} \mathrm{O}\left(\mathrm{CF}_{2} \mathrm{O}\right)_{n}\left(\mathrm{CF}_{2} \mathrm{CF}_{2} \mathrm{O}\right)_{m}\left(\mathrm{OCF}_{2} \mathrm{CF}_{2} \mathrm{O}\right)_{l} \mathrm{CF}_{3}$ with $n: m: l=65.8: 3.1: 0.2$ and a molecular weight 4883 . The measured total neutron cross section for this polymer yields the following parameters for Eq. (14): $\sigma_{0}=(1.53 \pm 2.2) \mathrm{b} / \mathrm{atom}, a=(8.8 \pm 0.5) \times 10^{-2} \mathrm{~b} /($ atom $\AA$ ) at $300 \mathrm{~K}$, and $\sigma_{0}=(-3 \pm 3) \mathrm{b} /$ atom, $a=(4.2 \pm 0.5) \times 10^{-2} \mathrm{~b} /($ atom $\AA)$ at $90 \mathrm{~K}$.

Fig. 7 shows the total cross section for polyethylene film. No visible deviation from $1 / v$ law is observed at the degree of precision of the test measurements, but our tentative measurement of the differential cross section reveals certain elastic scattering from density inhomogeneities even for such a material with small

\footnotetext{
${ }^{2}$ The investigated substances were produced by the Perm' branch of the Russian Scientific Center "Applied Chemistry"
} 
strength of coherent scattering $(\mathrm{U} \approx-9 \mathrm{neV})$. The fit of the total cross section data in accordance with Eq. (14) provides: $\sigma_{0}=(96 \pm 18) \mathrm{b}, \mathrm{a}=(2.07 \pm 0.04) \mathrm{b} /($ atom $\AA)$. From the measurements, it follows that with account of the neutron capture, extrapolated to the thermal point UCN upscattering contribution is $\sigma_{\text {ups, extr }}=$ $(2.07 \pm 0.4) \times 1.8-(0.334 \times 2 / 3)=(3.5 \pm 0.08)$ b/atom of the $\left(\mathrm{CH}_{2}\right)$-complex.

Fig. 8 presents the example of the measured differential cross section for copper.

The parameters of sample inhomogeneity appeared to differ from the parameters obtained from the total cross section: $\mathrm{Cu}$ (Gaussian): $G_{0}=(18 \pm 4) n e V^{2}, \rho_{0}=(420 \pm 45) \AA$. Analysis of more sophisticated models describing density fluctuations in inhomogeneous media calls for a more comprehensive investigation, which is beyond the scope of this test study.

\section{Acqnowledgments}

The spectrometer test and measurements were performed in the course of experiments 3-14-110, 3-14-132, and 3-14-152 at the PF2-test channel of the UCN turbine source of the High Flux Reactor at the Institut Laue-Langevin (Grenoble, France). We are grateful to the ILL reactor personal.

\section{References}

[1] F. L. Shapiro, In: Proceedings of the International Conference on Nuclear Structure with Neutrons, Budapest, 1972 edited by J. Ero and J. Szucs (Plenum, New York, 1972), p.259; A. Steyerl, in Neutron Physics, Springer Tracts in Modern Physics, 80, (Springer, Berlin, Heidelberg, New York, 1977), p. 57; R. Golub and J. M. Pendlebury, Rep. Progr. Phys., 42 (1979) 439; V. K. Ignatovich, Fizika ultrakholodnykh neitronov, (Nauka, Moscow,1986, in Russian); The Physics of Ultracold Neutrons, (Clarendon, Oxford, 1990); R. Golub, D. J. Richardson and S. Lamoreaux, Ultracold Neutrons (Adam Hilger, Bristol, 1991); J. M. Pendlebury, Ann. Revs. Nucl. Part. Sci., 43 (1993) 687.

[2] Proceedings of the International Conference on Fundamental Physics with slow Neutrons, Grenoble, 1998, Nucl. Instr. Meth., A440 (2000)

Proceedings of the International Conference on Precision Measurements with slow Neutrons, Gaithersburg, USA, 2004, Journ. of Res. NIST, 110, (2005) No. 3 and 4.

Proceedings of the International Workshop on Particle Physics with slow Neutrons, ILL, Grenoble, France, 2008, Nucl. Instr. Meth., A 611 (2009) Iss. 2-3.

[3] R. Golub, Revs. Mod. Phys. 68 (1996) 329.

[4] A. Michaudon, Los Alamos Preprint LA-13197-MS, Los Alamos, 1997.

[5] A. Steyerl, H. Nagel, F.-X. Schreiber, K.-A. Steinhauser, R. Gahler, W. Gläser, P. Ageron, J.-M. Astruc, N. Drexel, R. Gervais, W. Mampe, Phys. Lett., A116 (1986) 347.

[6] P. E. Hill, J. M. Anaya, T. J. Bowles, G. L. Greene, G. Hogan, S. Lamoreaux, L. Marek, R. Mortenson, C. L. Morris, A. Saunders, S. J. Seestrom, W. Teasdale, S. Hoedl, C.-Y. Liu, D. A. Smith, A. Young, B. W. Fillipone, J. Hua, T. Ito, E. Pasyuk, P. Geltenbort, A. Garcia, B. Fujikawa, S. Baessler, A. Serebrov, Nucl. Instr. Meth., A440 (2000) 674.

A. Saunders, J. M. Anaya, T. J. Bowles, B. W. Fillipone, P. Geltenbort, P. E. Hill, M.Hino, S. Hoedl, G. Hogan, T. Ito, K.W. Jones, T. Kawai, K. Kirch, S. Lamoreaux, C.-Y. Liu, M. Makela, L.J. Marek, J.W. Martin, C. L. Morris, R.N. Mortenson, A. Pichlmaier, S.J. Seestrom, A. Serebrov, D. Smith, W. Teasdale, B. Tipton, R.B. Vogelaar, A.R. Young, J. Yuan, Phys. Lett., B593 (2004) 55. 
[7] U. Trinks, F. J. Hartmann, S. Paul, W. Schott, Nucl. Instr. Meth., 440 (2000) 666.

[8] Workshop on PSI UCN Source, 17-18 Dec. 2001, PSI, Villigen, Switzerland; http://ucn.web.psi.ch/techrev_ucn/htm

[9] I.S. Altarev, Yu.V. Borisov, A.B. Brandin et al., Phys. Lett., 80A (1980) 413.

R. Golub, K. Böning K, Z. Phys. B51 (1983) 95;

Z.Ch. Yu , S.S. Malik, R. Golub, Z. Phys. B62 (1986) 137;

I.S. Altarev, V.A. Mityukhlyaev, A.P. Serebrov, A.A. Zakharov, Journ. Neutr. Res., 1 (1993) 71.

A.P. Serebrov, Nucl. Instr. Meth. A440 (2000) 653;

A. Serebrov A., A. Mityukhlyaev, A. Zakharov et al. Nucl. Instr. Meth. A440 (2000) 658.

[10] M.G.D. van der Grinten, J.M. Pendlebury, D. Shiers, Nucl. Instr. Meth. A423 (1999) 421;

F. Atchison, B. Blau, M. Daum et al., Phys. Lett. B642 (2006) 24;

F. Atchison, B. Blau, M. Daum, et. al., Nucl. Instr. Meth. B260 (2006) 647;

I. Altarev, M. Daum, A. Frei et al., Eur. Phys. Journ. A37 (2008) 9.

[11] M.I. Novopoltsev, Yu.N. Pokotilovski, JINR Commun. 3-81-828, 1981, Dubna;

M.I. Novopoltsev, Yu.N. Pokotilovski, I.G. Shelkova, Nucl. Instr. Meth, A264 (1988) 518;

M.I. Novopoltsev, Yu.N. Pokotilovski, Prib. Tekhn. Exper., no. 5 (2010) 19 [Instrum. Exp. Tekhn. 53 (2010) 635]; arXive: 1008.1419.

[12] A. Steyerl, Phys. Lett. 29B (1969) 33;

A. Steyerl and H. Vonach, Z. Phys. 250 (1972) 166;

A Steyerl, Nucl. Instr. Meth. 101 (1972), 295.

[13] A.V. Antonov, A.V. Isakov, S.P Kuznetsov et. al., Fiz. Tverd. Tela 26 (1984) 1585.

[14] Yu.Yu. Kosvintsev, Yu.A. Kushnir, V.I. Morozov, ZhETF 77 (1979) 1277 [JETP 50 (1979) 642];

A. Steyerl, S.S. Malik, P. Geltenbort et al., J. Phys., III, France, 7 (1997) 1941;

T. Bestle, P. Geltenbort, H. Just, Phys. Lett. A244 (1998) 217;

L.N. Bondarenko, P. Geltenbort, E. Korobkina et. al, Pis'ma ZhETF 68 (1998) 663;

P. Geltenbort, V.V. Nesvizhevsky, D.G. Kartashov et.al., Pis'ma ZhETF 70 (1999) 175;

V.V. Nesvizhevsky, A.V. Strelkov, P. Geltenbort, P. Iaydjiev, Eur. Phys. Journ. AP6 (1999) 151;

E.V. Lychagin, A.Y. Muzychka, V.V. Nesvizhevsky et al., Phys. Lett. B479 (2000) 353;

L.N. Bondarenko, P. Geltenbort, E. Korobkina et. al., Jad. Fiz., 65 (2002) 13.

E.V. Lychagin, D.G. Kartashov, A.Y. Muzychka, et al, Jad. Fiz. 65 (2002) 2052.

[15] D.J. Richardson, J.M. Pendlebury, P. Iaydjiev, Nucl. Instr. Meth. A308 (1991) 568.

[16] J.C. Bates, Phys. Lett. 88A (1882) 427; Nucl. Instr. Meth. A216 (1983) 535;

P. Ageron, W. Mampe, J.C. Bates, and J.M. Pendlebury, Nucl. Instr. Meth. A249 (1986) 261;

W. Mampe, P. Ageron, J.C. Bates et al., Nucl. Instr. Meth. A284 (1989) 111; Phys. Rev. Lett. 63 (1989) 593;

Yu.N. Pokotilovski, Nucl. Instr. Meth. A425 (1999) 320; 
S. Arzumanov, L. Bondarenko, S. Chernyavsky et al., Phys. Lett. B483 (2000) 15;

A. Pichlmaier, J. Butterworth, P. Geltenbort et al., Nucl. Instr. Meth. A440 (2000) 517; A. Pichlmaier, Dissertation. (TU München, 1999);

Yu.N. Pokotilovski, ZhETF 123 (2003) 203 [JETP 96 (2003) 172];

A. Serebrov, V. Varlamov, A. Kharitonov et al., Phys. Lett. B605 (2005) 72, Phys. Rev. C78 (2008) 035505;

Yu.N. Pokotilovski, I. Natkaniec, K. Holderna-Natkaniec, Physica B403 (2008) 1942.

[17] C.A. Baker, K. Green, M.G.D. van der Grinten et al., Nucl. Instr. Meth. A487 (2002) 511;

C.A. Baker, S.N. Balashov, K. Green et al., Nucl. Instr. Meth. A501 (2003) 517;

T. Kitagaki, K. Sakai, M. Hino et al., Nucl. Instr. Meth. A529 (2004) 425;

M. Lasakov, A. Serebrov, A. Khusainov et al., Journ. Res. Natl. Inst. Technol. 110 (2005) 289.

[18] M. Lengsfeld and A. Steyerl., Z.Phys. B27 (1977) 117;

A. Lermer, A. Steyerl, Phys. Stat. Sol. A33 (1976) 531.

[19] A. Steyerl, Proc of the 2nd Intern. School on Neutron Physics, Alushta, 1974, JINR, Dubna, D3-7991, 42; Springer, "Very Low Energy Neutrons", Springer Tracts in Modern Physics, Berlin, Heidelberg, N-Y.: Springer, 80 (1977) 57;

A.V. Stepanov, Fiz. Elem. Chast. i Atom. Jad. 7 (1976) 989.

[20] V.G. Grinev, A.I. Isakov, S.P. Kuznetsov et. al., Journ. Mosc. Phys. Soc., 2 (1992) 243.

[21] G.J. Cuello, J.R. Santisteban, R.E. Mayor et al., Nucl. Instr. Meth., A357 (1995) 519.

[22] A.K. Freund, Nucl. Instr. Meth. 213 (1983) 495.

[23] S.F. Mughabghab, M. Divadeenam, N.F. Nolte, Cross Sections Vol.1 Part A. Academic Prees INC. 1981; S.F. Mughabghab, Cross Sections Vol.1 Part B. Academic Press INC. 1984. 